\title{
Técnicas de umbralización para el procesamiento digital de imágenes de GEM- Foils
}

\author{
Thresholding techniques for digital image processing of GEM-Foils
}

\author{
Nayid Triana, Andrés E. Jaramillo, Rafael M. Gutiérrez, César A. Rodríguez \\ Ingeniería electrónica, Universidad Antonio Nariño, Bogotá, Colombia \\ natriana@uan. edu.co \\ andres.jaramillo@uan.edu.co \\ cesararodriguez@uan.edu.co
}

\begin{abstract}
Resumen- Una GEM-Foil (Gas Electron Multiplier - Foil) es un componente básico de nuevos detectores de radiación usados principalmente en experimentos de física de altas energías y física nuclear. El desempeño óptimo de tales detectores depende críticamente de la calidad de fabricación de dicho componente, ya que cualquier irregularidad en una de los cientos de miles de micro-perforaciones de la placa puede inducir una chispa durante el funcionamiento y dañar el detector. Actualmente, para evaluar la calidad de fabricación de una GEM-Foil se adquieren imágenes de alta resolución mediante sistemas ópticos que las digitalizan para ser luego examinadas por inspección visual. En este trabajo se comparan varias técnicas para realizar la binarización de las imágenes de las GEM-Foils, aplicando técnicas de umbralización global y local, con el propósito de mejorar la rapidez y eficiencia del procesamiento de las imágenes de las GEM-Foils, para realizar posteriormente su control de calidad. A partir de las pruebas realizadas se encontró que, mediante cálculos estadísticos simples, como el promedio del máximo y mínimo de intensidad, aplicando umbralización local adaptativa es posible realizar la segmentación de las imágenes de una manera eficiente sin tener que recurrir a métodos más complejos los cuales pueden implicar tiempos de proceso demasiado grandes.
\end{abstract}

Palabras clave- Detectores gaseosos, GEM, Placas de detectores, Segmentación de la imagen, Umbralización.

Abstract-A GEM-Foil (Gas Electron Multiplier - Foil) is a basic component of new radiation detectors used mainly in high energy physics and nuclear physics experiments. Optimum performance of such detectors depends critically on the quality of manufacture of such component, because any irregularity in one of the hundreds of thousands of micro-perforations in the plate can induce a spark during operation and damage the detector. Currently, in order to assess the quality of a GEM-Foil, high resolution images are acquired through optical systems to be digitized and then examined by visual inspection. In this work, various techniques to perform binarization of the images of the GEM-Foils are compared, by applying techniques of global and local thresholding, in order to improve the speed and efficiency of processing the images of the GEM-Foils for its quality control. From tests it was found that by simple statistical calculations as the average of the maximum and minimum intensity, using local adaptive thresholding is possible to carry out the segmentation of images in an efficient manner without having to use more complex methods which may involve too large process time.

Key Word - Gas detectors, GEM, Foils, Image segmentation, Thresholding.

\section{INTRODUCCIÓN}

Una GEM-Foil (del inglés Gas Electron Multiplier Foil) es una placa microperforada que actúa como elemento de amplificación de cargas eléctricas para uso en detectores de partículas por medio de la ionización de gases [1], la cual está permitiendo la exploración de nuevas aplicaciones en diferentes campos como la física de altas energías [2, 3], la física médica [4] y la astronomía [5], entre otras. En la figura 1 se muestra una vista en sección transversal y una vista plana de una GEM-Foil. Una GEM-Foil es una placa con micro agujeros distribuidos según un patrón definido, cuyos contornos corresponden a las perforaciones de las capas de cobre superior e inferior y la capa de un dieléctrico llamado Kapton, que se encuentra entre las dos capas de cobre. En la vista plana, se puede apreciar que debido a la estructura física de la GEM-Foil y a las técnicas usadas para obtener sus imágenes, en escalas de grises, los agujeros se ven de color gris claro y los contornos de Kapton se ven como anillos de color gris oscuro. El diseño y la fabricación de una GEM-Foil se realizan para obtener agujeros con un diámetro $d$ de $50 \mu m$, un diámetro externo $D$ de los anillos de Kapton de $70 \mu m$ y una distancia entre los centros de los agujeros $P$ de $140 \mu m$, como se muestra en la figura 1 . Se obtienen aproximadamente 600.000 agujeros para una GEMFoil de $10 \mathrm{~cm} \times 10 \mathrm{~cm} \mathrm{[6].}$ 


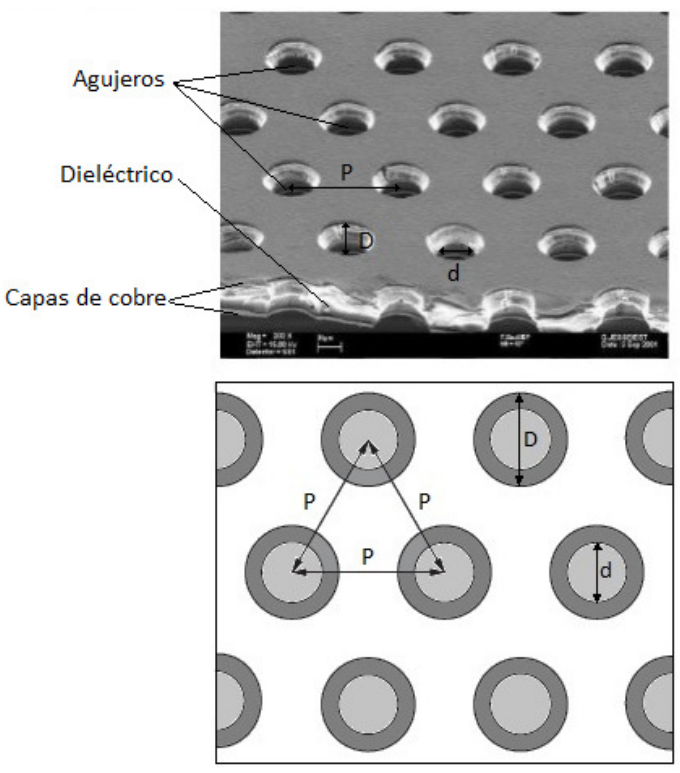

Figura 1. Ilustración de una GEM-Foil: imagen obtenida con microscopio electrónico de una GEM-Foil con vista de la sección transversal (arriba) [7], y diseño ideal del plano de la GEM-Foil (abajo).

Diferentes estudios experimentales y numéricos han mostrado que el desempeño de una GEM-Foil depende críticamente de las características geométricas de las perforaciones [8]. Una irregularidad en una de las cerca de 600.000 perforaciones puede inducir la descarga de una chispa, dañando la GEM-Foil; y en general, los defectos pueden alterar el funcionamiento del detector y por lo tanto la calidad y precisión de la señal detectada. Por lo tanto, se hace necesario desarrollar métodos de control de calidad de las GEM-Foils antes de ser instaladas en los detectores. Durante la última década se han presentado algunos trabajos para llevar a cabo este control de calidad [6], [9]. Sin embargo, estas técnicas pueden fracasar cuando no se provee una intensidad de luz homogénea sobre la GEM-Foil durante la adquisición, lo que causa distintos niveles de brillo de las imágenes. Además, el deterioro de la GEM-Foil por suciedad, desgaste o simplemente si las condiciones en que se han capturado las imágenes no resultan óptimas, pueden influir en el desempeño de tales métodos.

En este trabajo, se estudian algunos métodos de umbralización global y local con el objetivo de mejorar la rapidez y eficacia de la segmentación de imágenes de las GEM-Foils como parte esencial de los procesos de análisis de imágenes desarrollados para su control de calidad, especialmente en imágenes con presencia de anillos difíciles de detectar por inspección visual.

A partir de las pruebas realizadas se encontró que, mediante cálculos estadísticos simples, como el promedio del máximo y mínimo de intensidad, aplicando umbralización local adaptativa es posible realizar la segmentación de las imágenes de una manera eficiente sin tener que recurrir a métodos más complejos que pueden implicar tiempos de proceso demasiado grandes.

\section{MATERIALES Y MÉTODOS}

A. Imágenes de las GEM-Foils.

Para este trabajo se utilizaron imágenes de alta resolución obtenidas por Helsinki Institute of Physics (HIP) de una GEMFoil fabricada por Technology Transfer Agency (TECHTRA) y proporcionadas por Gesellschaft für Schwerionenforschung (GSI) dentro del programa de cooperación RD51-CERN [10]. En total se analizaron 432 imágenes en formato .PNG de una GEM-Foil de $10 \mathrm{~cm} \times 10 \mathrm{~cm}$. Cada imagen tiene un tamaño del orden de $23 \mathrm{MB}$, resultando aproximadamente $10 \mathrm{~GB}$ de información para procesar. La resolución de cada imagen es $3488 \times 2616$ pixeles y cada pixel tiene un tamaño de $1,7 \mu m \times$ $1,7 \mu \mathrm{m}$, lo que equivale a área total de $0,6 \mathrm{~cm} \times 0,45 \mathrm{~cm}$ por imagen.

\section{B. Pixel.}

El pixel es una unidad básica que permite representar un punto $p$ localizado en las coordenadas $r$ y $c$ con información $I(p)=$ $I(r, c)$ sobre la intensidad del punto, la cual va desde 0 hasta $L$ [11]. Usualmente un pixel requiere 8 bits para almacenar su intensidad $I(r, c)$, permitiendo una escala de $L=0$ hasta $L=$ 255 posibles valores de gris. La información del pixel se puede representar como

$$
\text { Pixel }=[p, I(p)]=[(r, c), I(r, c)]
$$

Donde

$$
I(r, c) \in\{0,1,2 \ldots L\}
$$

Las coordenadas $r$ y $c$, respectivamente, representan la fila y la columna donde se encuentra el elemento de la imagen de la GEM-Foil con intensidad $I(r, c)$. Si $I(p)$ es solo un número, entonces se dice que el pixel es monocromático, pero si es a color se describe como un vector de 3 elementos (Figura 2).

\section{Imágenes de la GEM-Foil en escala de gris.}

Las imágenes de la GEM-Foil se convirtieron a escala de grises aplicando la función rgb2gray () de MATLAB® (R2014a). Cada imagen en escala de gris $I$ es un arreglo matricial bidimensional de $N$ filas por $M$ columnas de pixeles $[p, I(p)]$ de información [11] (Figura 3), donde

$$
I(r, c) \in\{0,1,2 \ldots L\}
$$

con

$$
r=1,2, \ldots N \quad c=1,2, \ldots M \text {. }
$$

La expresión matricial de la imagen viene dada por 


$$
I=\left[\begin{array}{cccc}
I(1,1) & I(1,2) & \cdots & I(1, M) \\
I(2,1) & I(2,2) & \cdots & I(2, M) \\
\vdots & \vdots & \cdots & \vdots \\
\vdots & \vdots & \cdots & \vdots \\
I(N, 1) & I(N, 1) & \cdots & I(N, M)
\end{array}\right]
$$

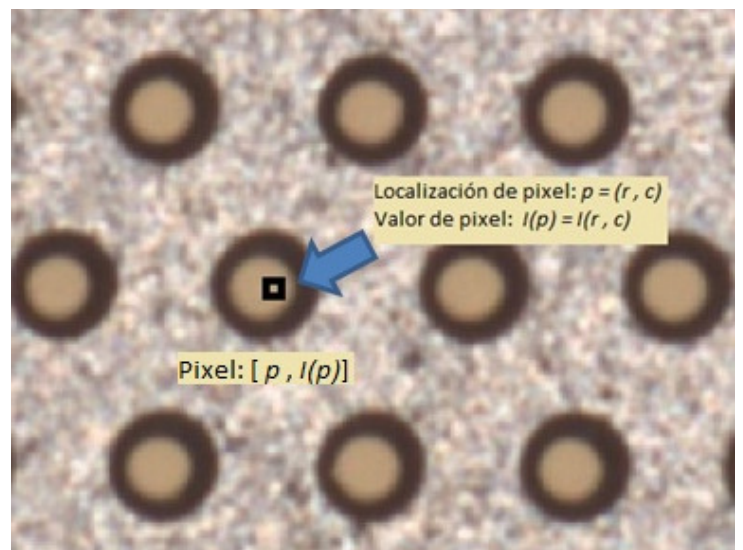

Figura 2. Pixel en una sección de imagen de una GEM-Foil fabricada por Technology Transfer Agency obtenida por Helsinki Institute of Physics y proporcionadas por Gesellschaft für Schwerionenforschung.

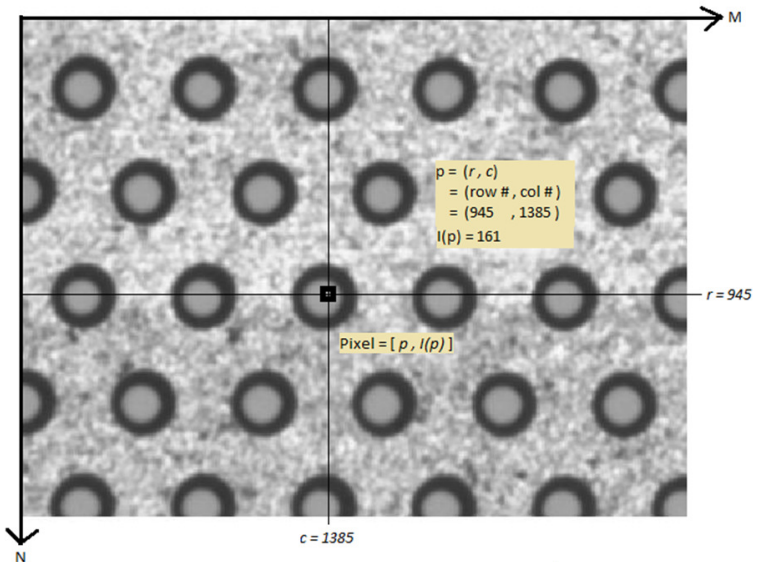

Figura 3. Sección de imagen de una GEM-Foil en escala de grises.

\section{Histograma de niveles de gris.}

El histograma $h_{I}$ de una imagen de la GEM-Foil representa gráficamente la cantidad $h_{I}(k)$ de pixeles con la misma intensidad $I(r, c)=k$, en el eje vertical, para cada valor de intensidad desde 0 hasta 255 en el eje horizontal [11]. La figura 4 muestra el histograma de una imagen de la GEM-Foil en escala de grises. Los picos representan la abundancia de pixeles de una cierta intensidad. Cómo se indica en la figura el pico agudo en la región de grises oscuros corresponde a los anillos de Kapton y el pico agudo a la derecha del histograma corresponde a los agujeros. Es importante resaltar que éste histograma corresponde a una imagen de buena calidad donde los agujeros se ven en general bastante bien; por eso la clara definición de los picos mencionados. En este trabajo se utilizó la función imhist () de MATLAB $®$ para generar el histograma directamente a partir de la imagen en niveles de gris.

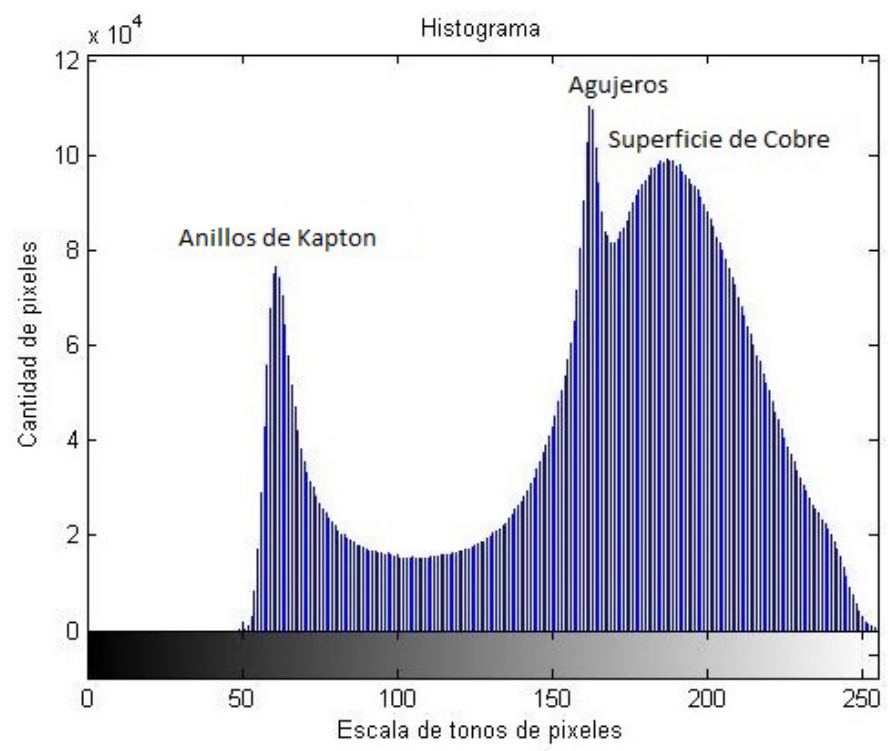

Figura 4. Histograma de una sección de una GEM-Foil en escala de grises.

E. Umbralización.

La umbralización es una técnica de segmentación simple y eficiente que permite separar los pixeles de una imagen en escala de grises en dos categorías a partir de un valor umbral de intensidad [11].

- Umbral global.

El umbral fijo o global, $T$, es aquel que es único sobre toda la imagen [12].

$$
b(r, c)=\left\{\begin{array}{l}
t_{0} \text { si } I(r, c)<T \\
t_{1} \text { si } I(r, c) \geq T
\end{array}\right.
$$

Donde $b(r, c)$ es la intensidad y $t_{0}$ y $t_{1}$ son los dos posibles niveles de gris del pixel umbralizado. Cuando el valor $t_{0}=0 \mathrm{y}$ el valor $t_{1}=1$ se dice que la imagen se ha binarizado. Esta estrategia puede resultar muy útil cuando se tiene una iluminación homogénea sobre toda la imagen y un histograma bimodal, como el que se muestra en la figura 5. 


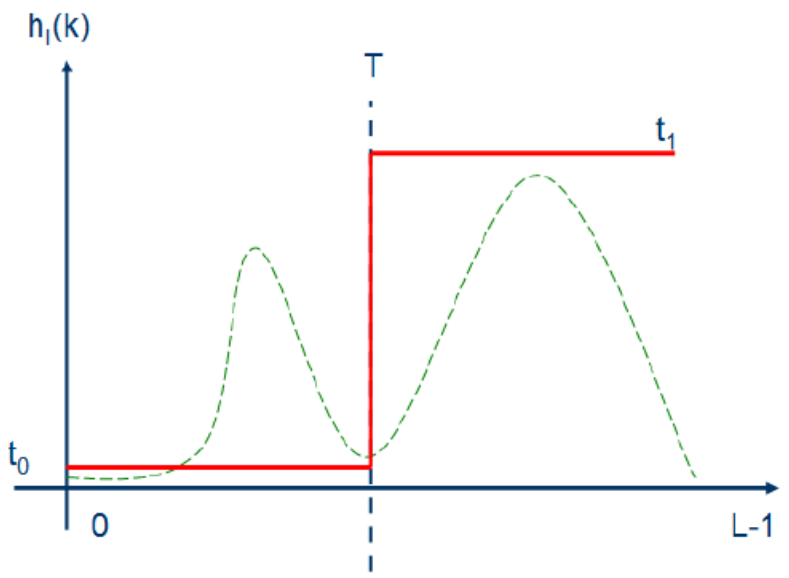

Figura 5. Histograma bimodal. $T$ es el valor de intensidad umbral y $t_{0}$ y $t_{1}$ son los dos posibles niveles de gris de los pixeles de la imagen umbralizada [11].

- Umbral local o adaptativo.

Cuando el histograma de una imagen de la GEM-Foil tiene una distribución no bimodal debido a los cambios de iluminación, como p.ej. en la figura 6 , se debe dividir la imagen en subimágenes para encontrar un umbral apropiado para cada una de ellas [12]. Dichas sub-imágenes pueden ser de diferentes formas y tamaños, lo que se define en el momento de implementar el algoritmo para la determinación del umbral local. De esta forma, no hay un único umbral para toda la imagen sino múltiples umbrales, uno para cada sub-imagen.

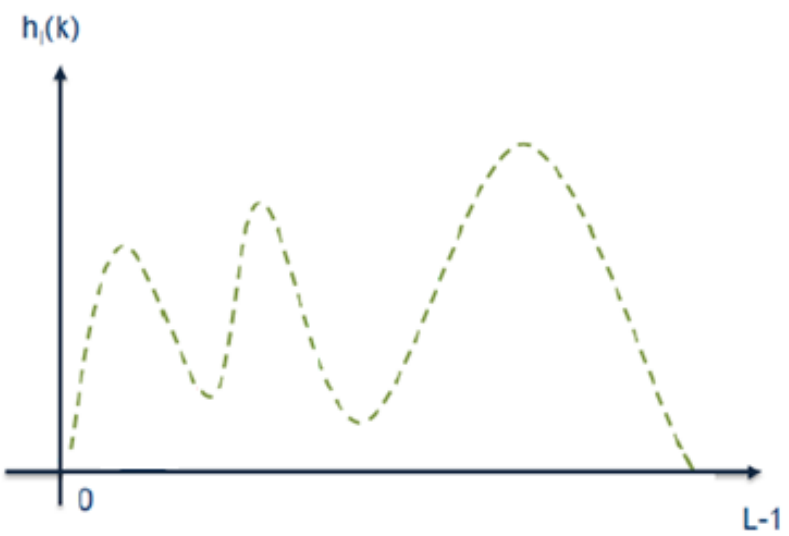

Figura 6. Histograma no bimodal [11].

F. Métodos de cálculo del umbral.

Las técnicas basadas en el histograma para la determinación del umbral más adecuado que permita segmentar los anillos de Kapton en las imágenes de las GEM-Foil resultan de gran utilidad ya que son fáciles de implementar, simples y generalmente rápidas.

Existen algunas medidas estadísticas básicas para la determinación del umbral a partir del histograma. Dentro de las cuales se encuentran la media, la mediana, y el promedio de máximo y mínimo. Aunque los métodos mediante la media y la mediana son simples, estos muestran resultados altamente ruidosos con respecto al método de máximo y mínimo cuando se trata con imágenes capturadas bajo diversas condiciones de iluminación [11].

Otras técnicas estadísticas más complejas como los algoritmos de Sauvola et al. [13], Niblack [14] y Papamarkos et al. [15], calculan el umbral de acuerdo a características locales espaciales y de intensidad. Estos métodos son tolerantes a los cambios de iluminación, pero son sensibles al ruido y, por lo tanto, degradan la imagen binarizada de la GEM-Foil [16].

Se realizaron pruebas con otros métodos de umbralización como el de Otsu [17] y el de la entropía [18]. Este último método también se descartó debido a los tiempos de proceso y el ruido en las imágenes binarias resultantes. Finalmente, por su rapidez y simplicidad, para este trabajo se seleccionaron 4 de los algoritmos de umbralización más reconocidos dentro del tratamiento digital de imágenes en la literatura científica, los cuales se describen a continuación:

- $\quad$ Promedio de máximo y mínimo.

Este método determina el valor medio entre la intensidad máxima y mínima del histograma [12],

$$
T=\frac{\max (I(r, c))+\min (I(r, c))}{2}
$$

\section{- Diferencia entre máximo y mínimo}

Esta técnica es similar al método del umbral mediante promedio de máximo y mínimo, pero en este caso se calcula el valor medio entre la diferencia de la intensidad máxima y mínima del histograma,

$$
T=\frac{\max (I(r, c))-\min (I(r, c))}{2}
$$

- $\quad$ Método de Otsu.

El método de Otsu es uno de los más populares en la literatura [17]. Este método considera que el histograma es bimodal y se itera a lo largo del histograma calculando para cada valor estimado posible de umbral la varianza de pesos dentro de cada clase o modo. El umbral óptimo se logra cuando la varianza entre clases genera un valor mínimo. El cálculo del umbral por el método de Otsu se realizó aplicando la función graythresh () de MATLAB®.

\section{- Mínimo local}

De la inspección visual del histograma en la figura 4, se puede ver que una opción para determinar el umbral que separe los anillos de Kapton del resto de la imagen es calcular el valor mínimo más bajo entre dos picos del histograma. Para realizar 
este cálculo, inicialmente se suaviza la curva aplicando la función smooth () de MATLAB $®$ hasta lograr una curva con 2 máximos locales. Luego, se calcula el umbral como valor mínimo entre los picos del histograma bimodal suavizado (Figura 7).
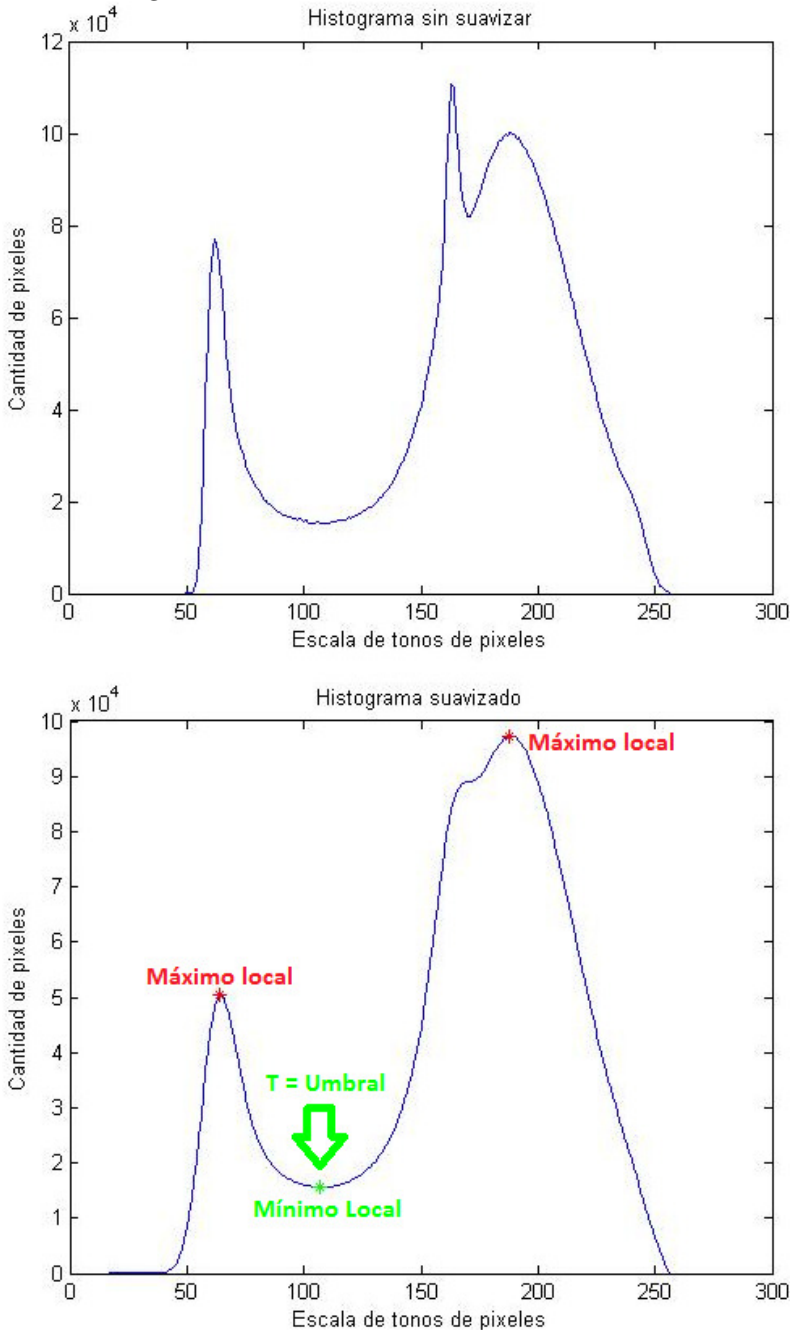

Figura 7. Curva envolvente del histograma de la figura 4, sin suavizar (arriba) y suavizado (abajo).

G. Comparación cualitativa y cuantitativa de los métodos de binarización.

Para la evaluación de cada uno de los algoritmos implementados en la binarización de las imágenes de la GEMFoil, se llevaron a cabo las siguientes etapas:

- $\quad$ Se generaron las imágenes binarizadas aplicando umbralización global con cada uno de los 4 métodos seleccionados y, por inspección visual, se comparó el ruido y los anillos de Kapton presentes en ellas.

- $\quad$ Se generaron las imágenes binarizadas aplicando umbralización local adaptativa para cada uno de los 4 métodos seleccionados, y se compararon con respecto a las imágenes binarizadas otenidas por umbralización global.

- $\quad$ Usando las funciones tic () y toc () de MATLAB $囚$, se midió el tiempo de ejecución en segundos que duró cada algoritmo en producir las 432 imágenes binarizadas de una GEM-Foil por umbralización global y local.

\section{RESULTADOS}

Para mostrar los resultados de la comparación entre la umbralización global y local de las imágenes de una GEM-Foil, con cada uno de los 4 métodos de binarización descritos en la sección anterior, se utilizó la imagen en escala de gris de la figura 8 , la cual tiene un tamaño de 3456 x 2560 pixeles, 1591 agujeros y niveles de brillo no homogéneos y una distribución no bimodal que se representa en la figura 9.

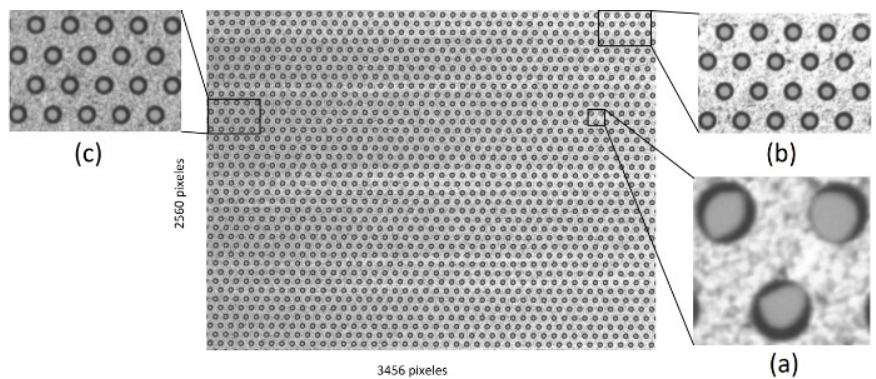

Figura 8. Imagen en escala de gris de una GEM-Foil fabricada por Technology Transfer Agency obtenida por Helsinki Institute of Physics y proporcionadas por Gesellschaft für Schwerionenforschung. Regiones ampliadas: (a) 3 anillos incompletos, (b) anillos en zona clara (brillo $=189)$ y $(\mathrm{c})$ anillos en zona oscura $($ brillo $=148$ ).

\section{- Umbralización global.}

Las figuras 10 y 11, ilustran los resultados de la umbralización global de las imágenes de la figura 8 para cada uno de los 4 métodos seleccionados. En la figura 10 se puede observar que el método para la determinación del umbral mediante promedio de máximo y mínimo (Figura 10d), presenta algunos problemas, ya que agrega más ruido a la imagen binarizada y genera anillos más gruesos. Por otro lado, el método para el cálculo del umbral por diferencia entre máximo y mínimo (Figura 10a) resulta el menos ruidoso seguido del método del mínimo local (Figura 10b), sin embargo, en ambos casos los anillos aparecen rotos. Además, a pesar que el método de Otsu (Figura 10c) es menos ruidoso que el método mediante promedio de máximo y mínimo (Figura 10d), con esta última técnica solamente aparece 1 anillo roto. 


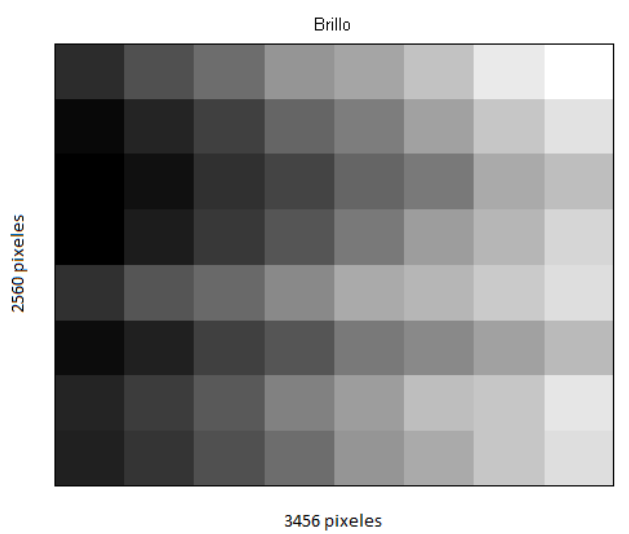

(a)

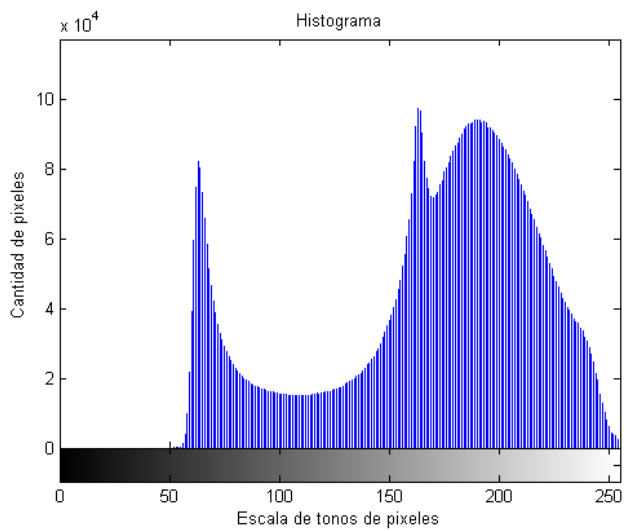

(b)

Figura 9. a) Imagen de niveles de brillo de la imagen de la figura 8 dividida en 64 secciones. b) Histograma no bimodal de la imagen de la figura 8.

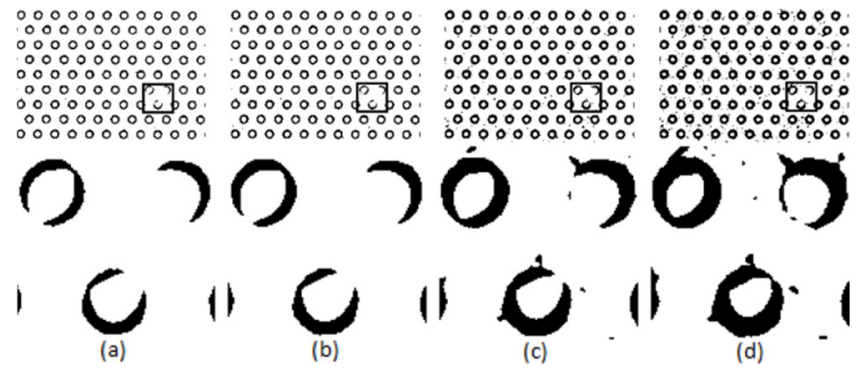

Figura 10. Binarización de imagen de la figura 8 por umbral global: (a) diferencia entre máximo y mínimo $(T=101.5)$;

(b) mínimo local $(T=110)$; (c) Otsu $(T=140)$; (d) promedio de máximo y mínimo $(T=153.5)$.

En la figura 11 se puede ver el resultado de la umbralización global en una zona oscura (Figura 8c), el cual es más ruidoso que en la zona clara (Figura 8b).

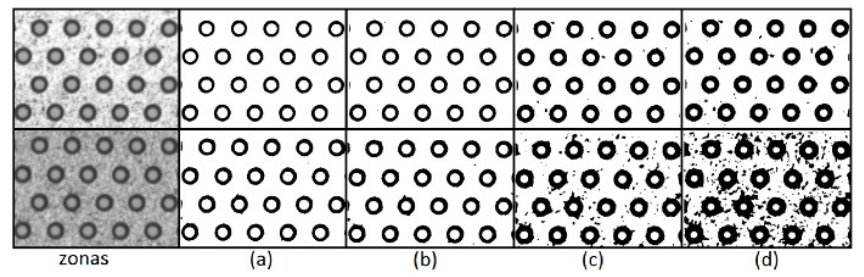

Figura 11. Binarización de la zona clara (fila superior) de la figura $8 \mathrm{~b}$, y la zona oscura (fila inferior) de la figura $8 \mathrm{c}$, por umbral global: (a) diferencia entre máximo y mínimo $(T=$ 101.5); (b) mínimo local ( $T=110)$; (c) Otsu $(T=140)$; (d) promedio de máximo y mínimo $(T=153.5)$.

\section{- Umbralización local}

Debido a que la mayoría de las imágenes utilizadas en este trabajo presentan variaciones de iluminación (Figura 9), se debió dividir cada imagen en sub-imágenes, como se indica en la figura 12, y encontrar un umbral local $T$ para cada una de esas sub-imágenes con cada uno de los 4 métodos de binarización usados en la umbralización global. La figura 13 muestra los resultados de la umbralización local en los anillos incompletos de la figura 8 a, después de dividir la imagen de la figura 8 en 16, 64, 256, 1024 y 4096 sub-imágenes y aplicar cada uno de los algoritmos de binarización a cada partición.
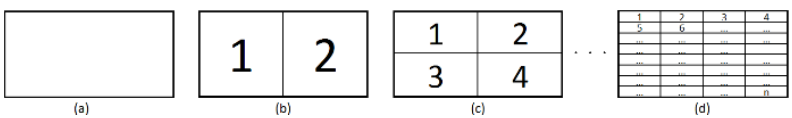

Figura 12. Representación de las particiones de una imagen: (a) la imagen inicial; (b) 2 sub-imágenes; (c) 4 sub-imágenes y (d) $n$ sub-imágenes.

En la figura 14 se puede observar que al aumentar el número de subdivisiones en la imagen se reduce el ruido y el grosor de los anillos encontrados en la zona oscura en comparación con los obtenidos por umbralización global que se muestran en la figura 11. Adicionalmente, en la figura 13 se puede ver que el método mediante promedio de máximo y mínimo en la umbralización local tuvo un mejor desempeño en relación con el mismo en la umbralización global que se muestra en la figura 10 , ya que completa los anillos después de dividir la imagen hasta sub-imágenes del tamaño de un anillo de $40 \times 54$ pixeles, esto es 4096 particiones. Por el contrario, con los métodos del mínimo local y Otsu se generan deformaciones de los anillos en algunos casos. 


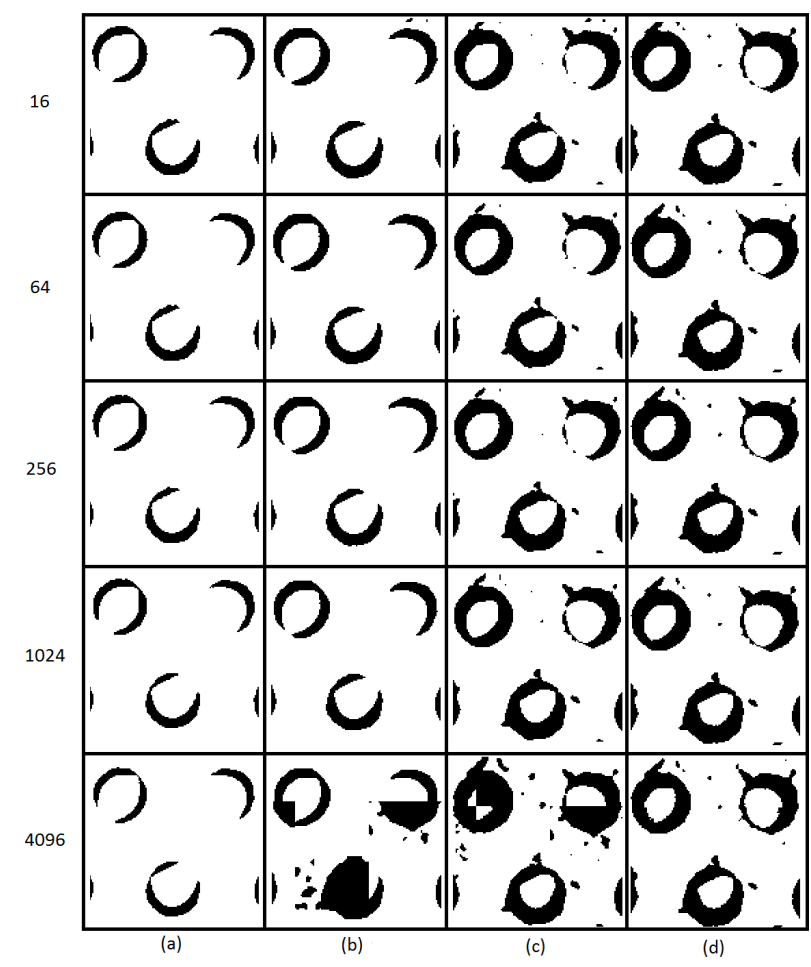

Figura 13. Binarización de la figura 8a con umbrales locales. De la columna izquierda a la derecha, métodos: (a) diferencia entre máximo y mínimo; (b) mínimo local; (c) Otsu; (d) promedio de máximo y mínimo. De la fila superior a la inferior, cantidad de subdivisiones: 16, 64, 256, 1024 y 4096.

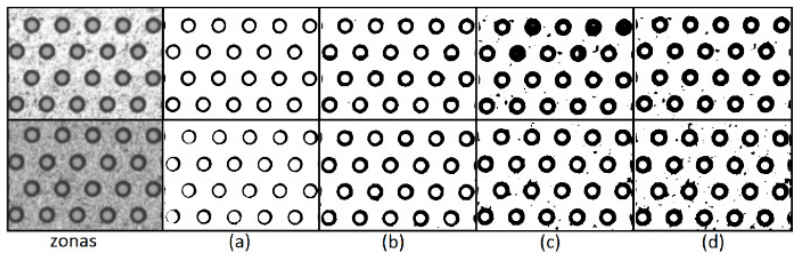

Figura 14. Binarización de la zona clara figura 8b (fila

superior), y oscura figura 8c (fila inferior) para 4096 subdivisiones con umbrales locales: (a) diferencia entre máximo y mínimo; (b) mínimo local; (c) Otsu; (d) promedio de máximo y mínimo.

- Tiempo de ejecución

La Tabla 1 muestra el tiempo total estimado que tardó cada algoritmo en binarizar las 432 imágenes de una GEM-Foil mediante umbralización global y con diferentes umbrales locales. En esta tabla se puede notar que los tiempos de ejecución de los algoritmos con diferentes umbrales locales se aumentan de forma creciente al aumentar el número de divisiones de la imagen. Además, para cualquier número de divisiones dado el algoritmo más lento fue el método del mínimo local.

\begin{tabular}{|c|c|c|c|c|c|c|}
\hline \multirow{2}{*}{ Método de umbralización } & \multicolumn{5}{|c|}{ Tiempo (en segundos) } \\
\cline { 2 - 7 } & Umbral & \multicolumn{5}{|c|}{ Umbrales locales (subdivisiones) } \\
\cline { 3 - 7 } & global & 16 & 64 & 256 & 1024 & 4096 \\
\hline Diferencia entre máximo y mínimo & 121,824 & 121,9104 & 124,4592 & 137,0736 & 155,9952 & 226,2384 \\
\hline Promedio de máximo y mínimo & 121,9536 & 122,0832 & 124,848 & 137,3328 & 156,4272 & 226,584 \\
\hline Otsu & 121,7376 & 122,6016 & 127,7856 & 145,4112 & 206,712 & 446,7744 \\
\hline Mínimo local & 122,3856 & 160,8336 & 288,576 & 832,4208 & 3015,7488 & 14712,3648 \\
\hline
\end{tabular}

Tabla 1. Comparativo de la rapidez en segundos de los métodos de binarización aplicados.

Estas pruebas fueron realizadas sobre una computadora HP ProDesk 400, Intel ${ }^{\circledR}$ Core $^{\mathrm{TM}}$ i5 de $3.20 \mathrm{GHz}$, RAM de $8 \mathrm{G}$, Sistema Operativo Windows 7 Professional de 64 bits, con MATLAB® (R2014a).

\section{CONCLUSIONES}

Este artículo presenta un análisis algunas de las técnicas más rápidas y eficientes de umbralización global y local para la binarización de imágenes de GEM-Foils. Teniendo en cuenta las diferencias en las características geométricas de los anillos de Kapton en las imágenes, los efectos de la binarización fueron analizados por inspección visual con el fin de comparar las diferentes técnicas de umbralización utilizadas.

Las pruebas realizadas muestran que el método de Otsu resulta ser el más rápido, pero sólo en el caso de la de umbralización global. En cambio, a medida que se aumenta el número de divisiones, en el caso de la umbralización local, los métodos de promedio y diferencia entre máximo y mínimo resultan ser más rápidos.

En general, se aprecia que debido a las diferencias locales de brillo que presentan las imágenes de las GEM-Foil, es necesario aplicar umbralización local adaptativa. Por otra parte, se encontró que las técnicas estadísticas simples como el promedio y la media entre máximos y mínimo ofrecen una solución eficiente para realizar la segmentación de los anillos de Kapton de dichas imágenes.

Específicamente, se pudo determinar que el método del promedio de máximo y mínimo fue el más eficaz completando los anillos cuando se realizan 4096 subdivisiones de la imagen. Sin embargo, en aquellos casos donde los anillos de Kapton se encuentren en zonas donde no hay grandes variaciones de brillo, es suficiente aplicar la umbralización adaptativa con menos subdivisiones lo cual implica un proceso más rápido y resultados con más bajo ruido. 


\section{REFERENCIAS}

[1]. B. Tamm, "Gas electron multipliers and a scanner for automated quality control", undergraduate Thesis, Massachusetts Institute of Technology, 2005.

[2]. C. Altunbas, M. Capéans, K. Dehmelt, J. Ehlers, J. Friedrich, I. Konorov, A. Gandi, S. Kappler, B. Ketzer, R. De Oliveira, S. Paul, A. Placci, L. Ropelewski, F. Sauli, F. Simon, and M. van Stenis, "Construction, test and commissioning of the triple-gem tracking detector for Compass", Nuclear Instruments and Methods in Physics Research A, vol. 490(1-2), pp.177-203, 2002.

[3]. B. Surrow, "The STAR Forward GEM Tracker", Nuclear Instruments and Methods in Physics Research A, vol. 617(1-3), pp. 196-198, 2010.

[4]. F. Anulli, A. Balla, G. Bencivenni, G. Corradi, C. D’Ambrosio, D. Domenici, G. Felici, M. Gatta, M.C. Morone, F. Murtas, and O. Schillaci, "A triple GEM gamma camera for medical application", Nuclear Instruments and Methods in Physics Research A, vol. 572, pp. 266-267, 2007.

[5]. H. Andersson, T. Andersson, J. Heino, J. Huovelin, K. Kurvinen, R. Lauhakangas, S. Nenonen, J. Ojala, R. Orava, J. Schultz, H. Sipilä, and O. Vilhu, "GEM detectors for X-ray astronomy", Nuclear Instruments and Methods in Physics Research A, vol. 513(1-2), pp. 155-158, 2003.

[6]. C. A. Rodríguez, R. M. Gutiérrez, A. E. Jaramillo, and N. Triana, "Morphological Analysis for Quality Assurance in GEM-Foils", in Proc. XX Symposium on Image, Signal Processing and Artificial Vision (STSIVA2015), pp. 1-7, 2015.

[7]. [Online] Available: http://gdd.web.cern.ch/GDD/ (Consultado el 13 de junio de 2016).

[8]. O. Bouianov, M. Bouianov, R. Orava, and V. Tikhonov, "Foil geometry effects on GEM characteristics", Nuclear Instruments and Methods in Physics Research A, vol. 458, pp. 698-701, 2001.

[9]. M. Kalliokoski, T. Hilden, F. Garcia, J. Heino, R. Lauhakangas, E. Tuominen, and R. Turpeinen, "Optical scanning system for quality control of GEM-Foils", Nuclear Instruments and Methods in Physics Research A, vol. 664, pp. 223-230, 2012.
[10]. [Online] Available: http://rd51public.web.cern.ch/RD51-Public/ (Consultado el 13 de junio de 2016).

[11]. J. A. Cortés, A. Muriel, and J. A. Mendoza, "Qualitative and quantitative Comparison of Basic histogram-based global Thresholding techniques for Digital Image Processing", Scientia et Technica, vol. 3(49), pp. 266-272, 2011.

[12]. R. Gonzalez and R. Woods, Digital Image Processing, N.J.: Prentice Hall, 2008.

[13]. J. Sauvola and M. Pietikainen, "Adaptive Document Image Binarization”, Pattern Recognition, vol. 33(2), pp. 225 236, 2000.

[14]. W. Niblack, An Introduction to Digital Image Processing, Englewood Cliffs, N.J.: Prentice Hall, pp.115-116, 1986.

[15]. N. Papamarkos and B. Gatos, "A new approach for multilevel threshold selection", Graphical Models and Image Processing, vol. 56(5), pp. 357-370, 1994.

[16]. V. Vonikakis, I. Andreadis, and N. Papamarkos, "Adaptive document binarization: A human vision approach" [Online] Available: http://robotics.pme.duth.gr /pubs /Conferences/ADAPTIVE\%20DOCUMENT\%20BINARIZA TION\%20A\%20human\%20vision\%20approach.pdf (Consultado el 13 de Junio de 2016).

[17]. N. Otsu, "A Threshold Selection Method from GrayLevel Histograms", IEEE Transactions on Systems, Man, and Cybernetics, Vol. 9, No. 1, pp. 62-66, 1979.

[18]. M. Chowdhury and W Little, "Image thresholding techniques", [Online] Available: http://ieeexplore.ieee.org/xpl/freeabs_all.jsp?arnumber=51959 9 (Consultado el 13 de Junio de 2016). 